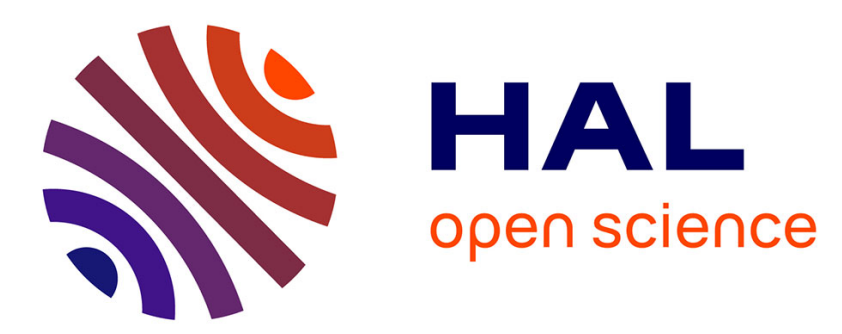

\title{
Design of Interval Observers for Estimation and Stabilization of Discrete-Time LPV Systems
}

Denis Efimov, Tarek Raissi, Wilfrid Perruquetti, Ali Zolghadri

\section{To cite this version:}

Denis Efimov, Tarek Raissi, Wilfrid Perruquetti, Ali Zolghadri. Design of Interval Observers for Estimation and Stabilization of Discrete-Time LPV Systems. IMA Journal of Mathematical Control and Information, 2015, pp.1-16. 10.1093/imamci/dnv023 . hal-01162680

\author{
HAL Id: hal-01162680 \\ https://hal.inria.fr/hal-01162680
}

Submitted on 11 Jun 2015

HAL is a multi-disciplinary open access archive for the deposit and dissemination of scientific research documents, whether they are published or not. The documents may come from teaching and research institutions in France or abroad, or from public or private research centers.
L'archive ouverte pluridisciplinaire HAL, est destinée au dépôt et à la diffusion de documents scientifiques de niveau recherche, publiés ou non, émanant des établissements d'enseignement et de recherche français ou étrangers, des laboratoires publics ou privés. 
IMA Journal of Mathematical Control and Information Page 1 of 16 doi:10.1093/imamci/dnnxxx

\title{
Design of Interval Observers for Estimation and Stabilization of Discrete-Time LPV Systems
}

\author{
Denis Efimov, Tarek Raïssi, Wilfrid Perruquetti, Ali Zolghadri \\ denis.efimov@inria.fr,tarek.raissi@cnam.fr,wilfrid.perruquetti@inria.fr, \\ ali.zolghadri@ims-bordeaux.fr
}

[Received on May 6, 2015]

\begin{abstract}
This work is devoted to interval observers design for discrete-time Linear Parameter-Varying (LPV) systems under the assumption that the vector of scheduling parameters is not available for measurements. Two problems are considered: a pure estimation problem and an output stabilizing feedback design problem where the stability conditions are expressed in terms of Linear Matrix Inequalities (LMIs). The efficiency of the proposed approach is demonstrated through computer simulations.
\end{abstract}

Keywords: Interval estimation, LPV systems, stabilization.

\section{Introduction}

Many nonlinear system models can be represented by (or transformed into) an LPV form. The main advantage is that the partial linearity of LPV models allows one to apply plenty of methods developed for linear systems $[35,22,34,36,19]$. In some situations, the vector of scheduling parameters of an LPV system is not available for measurements (partially or completely). For example, for an aircraft, LPV models are well situated to characterize physical nonlinear model derived from aerospace engineering. To generate LPV models, mass and center of gravity are usually used as scheduling parameters. Although these parameters can be available on-board (for example mass estimation based on fuel consumption), their values are relatively crude and should be considered to be an interval, rather than a single point measurement. In such situations, set-membership estimation techniques offer an appealing alternative solution where all variables are unknown but bounded.

Set-membership state estimation has been intensively studied during the past decades. Most of the techniques proposed in the literature are based on the well-known prediction/correction mechanism. The prediction consists in propagating the state set available at $t_{k}$ in order to predict an outer approximation at $t_{k+1}$, while the correction step uses the measurement available at $t_{k+1}$ to contract the predicted set. Depending on the system model (linear or nonlinear), the state set is wrapped into particular geometrical shapes such as ellipsoids [29], zonotopes [1] or intervals [15, 31]. For Linear Time-Invariant (LTI) systems, there exist several efficient solutions to perform state/estimation with guaranteed performances at a low computational cost (for instance by using ellipsoids, zonotopes, polytopes, ...). This is not the case for nonlinear / uncertain systems, where usually interval arithmetics is used. The advantage of interval analysis is that it is possible to propagate uncertainties for nonlinear and LPV systems. Nevertheless, due to wrapping and dependence effects (see for instance [16]), the stability analysis of these algorithms is complicated for systems subject to large uncertainties. In several works, the pessimism is reduced, at the cost of a computational burden, by using for instance sub-paving and constraint satisfaction techniques. An alternative approach is used in our paper, it is based on the so-called interval observers where two judicious conventional/pointwise observers are built in order to estimate, at each time, an outer approximation (a box) for the state. 
There are several approaches to design interval observers (or set-membership observers) [15, 18, 2, 28]. This paper continues the trend of interval observer design based on the monotone systems theory $[2,28,32,30,9]$. One of the most restrictive assumptions for interval observers design deals with cooperativity of the interval estimation error dynamics, which has been recently relaxed, for instance in $[23,30,6,24]$. Indeed, in [25, 24] (resp. in [23]), a constructive technique, which allows one to transform any Schur stable (resp. Hurwitz) matrix into a nonnegative Schur stable (resp. Metzler and Hurwitz) matrix, through a possibly time-varying change of coordinates, has been proposed. In $[30,11]$, it has been shown that under some conditions, the change of coordinates can be time-invariant. Furthermore, in order to design an interval observer for systems with non-constant matrices dependent on measurable input-output signals and time, an extension of the result from [30] has been presented in [11], which enables to calculate a constant similarity transformation matrix representing a given interval of matrices into an interval of Metzler matrices. Similar results for discrete-time systems have been recently obtained in $[24,10]$ (for the case of measured vector of scheduling parameters).

The problem becomes more challenging if a control design is required. There exists a few methods, which are able to stabilize an uncertain nonlinear system using only output measurements, and the LPV system approach is one of the most popular among them [20,27]. Interval observers can be used for the stabilization of a continuous-time LPV system (or a class of nonlinear uncertain systems) under assumption that the vector of scheduling parameters is not measured [12].

The contribution of this paper is considered to be twofold. Firstly, an extension of the results from [10] on interval observers design for discrete-time LPV systems with unmeasurable vector of scheduling parameters is proposed. Secondly, a stabilizing control design based on interval observers as in [12] is given. The proposed methodology consists in stabilizing the LPV system even when the scheduling parameter vector is unmeasurable. Since the interval observer satisfies $\underline{x}(t) \leqslant x(t) \leqslant \bar{x}(t), \forall t \geqslant t_{0}$, therefore, the stabilization of the bounds $\underline{x}$ and $\bar{x}$, considered as the outputs of the observer, ensures the same property for the LPV system state $x$.

The paper is organized as follows. Some basic facts from the theory of interval estimation are recalled in Section 2. Problem formulation and some preliminaries are presented in Section 3. The main result is described in Section 4, where an interval observer is designed and next it is shown how it can be used for stabilization. Convincing computer simulations are presented in Section 5.

\section{Preliminaries}

The sets of real and integer numbers are denoted by $\mathbb{R}$ and $\mathbb{N}$ respectively, $\mathbb{R}_{+}=\{\tau \in \mathbb{R}: \tau \geqslant 0\}$ and $\mathbb{N}_{+}=\mathbb{N} \cap \mathbb{R}_{+}$. Euclidean norm of a vector $x \in \mathbb{R}^{n}$ will be denoted by $|x|,|x|_{\infty}=\max _{1 \leqslant i \leqslant n}\left|x_{i}\right|$, and for a measurable and locally essentially bounded input $u: \mathbb{N} \rightarrow \mathbb{R}$ (we will use the convention $u(t)=u_{t}$ for a $\left.t \in \mathbb{N}_{+}\right)$the symbol $\|u\|_{\left[_{t_{0}, t_{1}}\right)}$ denotes its $\mathscr{L}_{\infty}$ norm $\|u\|_{\left.t_{0}, t_{1}\right)}=\sup \left\{\left|u_{t}\right|, t \in\left[t_{0}, t_{1}\right)\right\}$, $\|u\|=\|u\|_{[0,+\infty)}$. We will denote by $\mathscr{L}_{\infty}$ the set of all inputs $u$ with the property $\|u\|<\infty$. Denote the sequence of integers $1, \ldots, k$ by $\overline{1, k}$. The symbols $I_{n}, E_{n \times m}$ and $E_{p}$ denote the identity matrix with dimension $n \times n$, the matrix with all elements equal 1 with dimensions $n \times m$ and $p \times 1$ respectively. For a symmetric matrix $M \in \mathbb{R}^{n \times n}$, we denote by $\lambda(M)$ the vector of its eigenvalues, $\lambda_{\max }(M)=\max \lambda(M)$, $\lambda_{\min }(M)=\min \lambda(M)$ and by $\|A\|_{2}=\sqrt{\lambda_{\max }\left(A^{T} A\right)}$ the induced matrix norm for $A \in \mathbb{R}^{n \times n}$.

\subsection{Interval analysis}

For two vectors $x_{1}, x_{2} \in \mathbb{R}^{n}$ or matrices $A_{1}, A_{2} \in \mathbb{R}^{n \times n}$, the relations $x_{1} \leqslant x_{2}$ and $A_{1} \leqslant A_{2}$ are understood elementwise. The relation $P \prec 0(P \succeq 0)$ means that the matrix $P \in \mathbb{R}^{n \times n}$ is negative (positive semi-) 
definite. Given a matrix $A \in \mathbb{R}^{m \times n}$, define $A^{+}=\max \{0, A\}, A^{-}=A^{+}-A$ (similarly for vectors) and denote the matrix of absolute values of all elements by $|A|=A^{+}+A^{-}$.

LEMMA 2.1 Let $\underline{A} \leqslant A \leqslant \bar{A}$ for some $\underline{A}, \bar{A}, A \in \mathbb{R}^{n \times n}$ and $\underline{x} \leqslant x \leqslant \bar{x}$ for $\underline{x}, \bar{x}, x \in \mathbb{R}^{n}$, then

$$
\underline{A}^{+} \underline{x}^{+}-\bar{A} \underline{x}^{-}-\underline{A}^{-} \bar{x}^{+}+\bar{A}^{-} \bar{x}^{-} \leqslant A x \leqslant \bar{A}^{+} \bar{x}^{+}-\underline{A}^{+} \bar{x}^{-}-\bar{A} \underline{x}^{+}+\underline{A}^{-} \underline{x}^{-} .
$$

Proof. By definition $A x=\left(A^{+}-A^{-}\right)\left(x^{+}-x^{-}\right)=A^{+} x^{+}-A^{+} x^{-}-A^{-} x^{+}+A^{-} x^{-}$, where all terms are elementwise nonnegative, which gives the required relations.

\subsection{Cooperative discrete-time linear systems}

A matrix $A \in \mathbb{R}^{n \times n}$ is called Schur stable if all its eigenvalues have the norm less than one, it is called nonnegative if all its elements are nonnegative (i.e $A \geqslant 0$ ), and it is called Metzler if all its off-diagonal elements are nonnegative. Any solution of the system

$$
x_{t+1}=A x_{t}+\omega_{t}, \omega: \mathbb{N}_{+} \rightarrow \mathbb{R}_{+}^{n}, t \in \mathbb{N}_{+},
$$

with $x_{t} \in \mathbb{R}^{n}$ and a nonnegative matrix $A \in \mathbb{R}_{+}^{n \times n}$, is elementwise nonnegative for all $t \geqslant 0$ provided that $x(0) \geqslant 0$ [14]. Such a system is called cooperative (monotone) or nonnegative [14].

Lemma 2.2 [13] A matrix $A \in \mathbb{R}_{+}^{n \times n}$ is Schur stable iff there exists a diagonal matrix $D \in \mathbb{R}_{+}^{n \times n}$ such that $A^{T} D A-D \prec 0$.

In the sequel we are interested in a Luenberger-like observer design with the gain $L$ such that the matrix $A-L C$ (the closed loop matrix of the estimation error dynamics) is Schur stable and nonnegative. Usually it is not possible to find such a matrix $L$. However a change of variables $z(t)=S x(t)$ with a nonsingular matrix $S$ can be proposed such that, in the new coordinates, the matrix $S(A-L C) S^{-1}$ would satisfy the required properties. An idea how to design such a matrix $S$ is given in the lemma below.

LEMma 2.3 [30] Given the matrices $A \in \mathbb{R}^{n \times n}, R \in \mathbb{R}^{n \times n}$ and $C \in \mathbb{R}^{p \times n}$. If there exists a matrix $L \in \mathbb{R}^{n \times p}$ such that $\lambda(A-L C)=\lambda(R)$, and there exist vectors $\rho_{1} \in \mathbb{R}^{1 \times n}, \rho_{2} \in \mathbb{R}^{1 \times n}$ such that the pairs $\left(A-L C, \rho_{1}\right)$ and $\left(R, \rho_{2}\right)$ are observable, then there is a nonsingular $S \in \mathbb{R}^{n \times n}$ such that $R=S(A-$ $L C) S^{-1}$.

The main difficulty is to prove existence of a real and nonsingular matrix $S$, and to provide a constructive approach of its calculation. This result was used in [30] to design interval observers for continuous-time LTI systems with a Metzler matrix $R$, and in the work [10], Lemma 2.3 has been also applied to a nonnegative matrix $R$.

\section{Problem statement}

Consider an LPV system described by:

$$
\begin{aligned}
x_{t+1} & =\left[A_{0}+\Delta A\left(\rho_{t}\right)\right] x_{t}+B u_{t}+d_{t}, \\
y_{t} & =C x_{t}+v_{t}, t \in \mathbb{N}_{+},
\end{aligned}
$$

where $x_{t} \in \mathbb{R}^{n}$ is the state, $y_{t} \in \mathbb{R}^{p}$ is the output available for measurements, $u_{t} \in \mathbb{R}^{m}$ is the control, $\rho_{t} \in \Pi \subset \mathbb{R}^{r}$ is the vector of scheduling parameters with a known $\Pi, \rho \in \mathscr{L}_{\infty}^{r}$. The values of the scheduling vector $\rho$ are not available for measurements, and only the set of admissible values $\Pi$ is given. 
The matrices $A_{0} \in \mathbb{R}^{n \times n}, B \in \mathbb{R}^{n \times n}$ and $C \in \mathbb{R}^{p \times n}$ are known, the matrix function $\Delta A: \Pi \rightarrow \mathbb{R}^{n \times n}$ is piecewise continuous. The signals $d \in \mathscr{L}_{\infty}^{n}$ and $v \in \mathscr{L}_{\infty}^{p}$ are the exogenous disturbance and measurement noise respectively, the exact current values of $d_{t}$ and $v_{t}$ are not available. For brevity of presentation we will use the following assumptions in this work.

Assumption $3.1 \underline{d}_{t} \leqslant d_{t} \leqslant \bar{d}_{t}$ and $\left|v_{t}\right| \leqslant V$ for all $t \in \mathbb{N}_{+}$and for some known $\underline{d}, \bar{d} \in \mathscr{L}_{\infty}^{n}$ and $V \in \mathbb{R}_{+}$. Assumption $3.2 \underline{\Delta A} \leqslant \Delta A(\rho) \leqslant \overline{\Delta A}$ for all $\rho \in \Pi$ and some known $\underline{\Delta A}, \overline{\Delta A} \in \mathbb{R}^{n \times n}$.

Note that it is straightforward to compute $\underline{\Delta A}, \overline{\Delta A}$ for a given compact set $\Pi$ and a known function $\Delta A: \Pi \rightarrow \mathbb{R}^{n \times n}$ (in a polytopic case, for example).

An interval observer for a class of continuous-time LPV systems has been proposed in [12] in the framework of stabilization of LPV systems. In that work the stability of interval observers was ensured by a proper choice of control input providing the uncertain system stabilization. For the case of a measured vector $\rho_{t}$ in (3.1), an interval observer was proposed in [10] using the result of Lemma 2.3. Let us consider how to extend these results to a discrete-time LPV system (3.1) with unmeasured $\rho$.

Before introduction of the interval observer equations note that for a matrix $L \in \mathbb{R}^{n \times p}$, the system (3.1) can be rewritten as follows:

$$
\begin{aligned}
x_{t+1}= & {\left[A_{0}-L C\right] x_{t}+\Delta A\left(\rho_{t}\right) x_{t} } \\
& +L\left[y_{t}-v_{t}\right]+B u_{t}+d_{t},
\end{aligned}
$$

and according to Lemma 2.1 and Assumption 3.2:

$$
\begin{gathered}
\underline{\Delta A}^{+} \underline{\underline{x}}_{t}^{+}-\overline{\Delta A}{ }^{+} \underline{x}_{t}^{-}-\underline{\Delta A}{ }^{-} \bar{x}_{t}^{+}+\overline{\Delta A} \bar{x}_{t}^{-} \leqslant \Delta A\left(\rho_{t}\right) x_{t} \\
\leqslant \overline{\Delta A} \bar{x}_{t}^{+}-\underline{\Delta A^{+}} \bar{x}_{t}^{-}-\overline{\Delta A} \underline{x}_{t}^{+}+\underline{\Delta A}^{-} \underline{x}_{t}^{-}
\end{gathered}
$$

provided that $\underline{x}_{t} \leqslant x_{t} \leqslant \bar{x}_{t}$ for any $\underline{x}_{t}, \bar{x}_{t}, x_{t} \in \mathbb{R}^{n}, \rho_{t} \in \Pi$ and $t \in \mathbb{N}_{+}$.

The design of an interval observer can be considerably simplified for the case of nonnegative systems $x_{t} \in \mathbb{R}_{+}^{n}$ (which means that all the elements of $\left[A_{0}+\Delta A\left(\rho_{t}\right)\right]$ are nonnegative and $\left.B u_{t}+d(t) \geqslant 0, \forall t \geqslant t_{0}\right)$. Nevertheless, in this work we will consider a general case of non cooperative systems.

\section{Main results}

The proposed structure of interval observer for the system (3.1) is based on (3.2) and the relations (3.3):

$$
\begin{gathered}
\underline{x}_{t+1}=\left[A_{0}-\underline{L C}\right] \underline{x}_{t}+B u_{t}+\left[\underline{\Delta A}^{+} \underline{x}_{t}^{+}-\overline{\Delta A} \underline{x}_{t}^{-}\right. \\
\left.-\underline{\Delta A} \bar{x}_{t}^{+}+\overline{\Delta A}^{-} \bar{x}_{t}^{-}\right]+\underline{L} y_{t}-|\underline{L}| V E_{p}+\underline{d}_{t}, \\
\bar{x}_{t+1}=\left[A_{0}-\bar{L} C\right] \bar{x}_{t}+B u_{t}+\left[\overline{\Delta A} \bar{x}_{t}^{+}-\underline{\Delta A}{ }^{+} \bar{x}_{t}^{-}\right. \\
\left.-\overline{\Delta A}^{-} \underline{x}_{t}^{+}+\underline{\Delta A}^{-} \underline{x}_{t}^{-}\right]+\bar{L} y_{t}+|\bar{L}| V E_{p}+\bar{d}_{t},
\end{gathered}
$$

where $\underline{x}_{t}, \bar{x}_{t}$ are the lower ad upper interval estimates of $x_{t}, \underline{L} \in \mathbb{R}^{n \times p}$ and $\bar{L} \in \mathbb{R}^{n \times p}$ are the observer gains to be designed. For the sake of generality, two gains $\underline{L}$ and $\bar{L}$ are used while in [10], it was assumed that $\underline{L}=\bar{L}$. Note that due to the presence of $\underline{x}_{t}^{+}, \underline{x}_{t}^{-}, \bar{x}_{t}^{+}$and $\bar{x}_{t}^{-}$, the interval observer (4.1) is a globally Lipschitz nonlinear system.

Next, two cases are considered in this section. Firstly, assuming that the state $x_{t}$ is bounded and the control signal $u_{t}$ is given, then the goal is to design two gains $\underline{L}$ and $\bar{L}$ such that (4.1) ensures the 
interval estimation of $x_{t}$ (i.e. $\underline{x}_{t} \leqslant x_{t} \leqslant \bar{x}_{t}$ for all $t \in \mathbb{N}_{+}$) and boundedness of $\underline{x}_{t}, \bar{x}_{t}$. Secondly, the interval observer (4.1) with the prior designed gains $\underline{L}, \bar{L}$ is used to compute a stabilizing control law $u_{t}=U\left(\underline{x}_{t}, \bar{x}_{t}, y_{t}\right)$ guaranteeing an interval estimation of the state $x_{t}$ and its boundedness together with $\underline{x}_{t}$, $\bar{x}_{t}$.

\subsection{Interval estimation}

The conditions, which have to be imposed on $\underline{L}, \bar{L}$ in order to ensure an interval estimation of $x_{t}$ and boundedness of $\underline{x}_{t}, \bar{x}_{t}$, are formulated in theorem 4.1.

Assumption 4.1 There exist $\underline{L}$ and $\bar{L}$ satisfying:

$$
A_{0}-\underline{L} C, A_{0}-\bar{L} C \in \mathbb{R}_{+}^{n \times n} .
$$

THEOREM 4.1 Let assumptions 3.1, 3.2 and 4.1 be satisfied, $x \in \mathscr{L}_{\infty}^{n}$ and $u \in \mathscr{L}_{\infty}^{m}$. Then, the relations

$$
\underline{x}_{t} \leqslant x_{t} \leqslant \bar{x}_{t} \quad \forall t \in \mathbb{N}_{+}
$$

are satisfied provided that $\underline{x}_{0} \leqslant x_{0} \leqslant \bar{x}_{0}$. In addition, if there exist a diagonal matrix $P \in \mathbb{R}^{2 n \times 2 n}, P \succ 0$, matrices $Q \in \mathbb{R}^{2 n \times 2 n}, Q=Q^{T} \succ 0, H \in \mathbb{R}^{2 n \times 2 n}, H=H^{T} \succ 0$ and a constant $\gamma>0$ such that the following matrix inequality is verified

$$
\begin{gathered}
\Phi=\left[\begin{array}{ccc}
\Psi & G^{T} P & G^{T} P \\
P G & P-\gamma I_{2 n} & P \\
P G & P & P-H
\end{array}\right] \preceq 0, \\
\Psi=G^{T} P G-P+Q+\gamma \eta^{2} I_{2 n}, \\
G=\left[\begin{array}{cc}
A_{0}-\underline{L} C+\underline{\Delta A^{+}} & 0 \\
0 & A_{0}-\bar{L} C+\overline{\Delta A}^{+}
\end{array}\right],
\end{gathered}
$$

where $\eta=\sqrt{2}\left(\left\|\underline{\Delta A^{+}}-\overline{\Delta A}^{+}\right\|_{2}+\left\|\underline{\Delta A^{-}}\right\|_{2}+\|\overline{\Delta A}\|_{2}\right)$, then $\underline{x}, \bar{x} \in \mathscr{L}_{\infty}^{n}$.

Proof. Consider the dynamics of interval estimation errors $\underline{e}_{t}=x_{t}-\underline{x}_{t}$ and $\bar{e}_{t}=\bar{x}_{t}-x_{t}$ :

$$
\begin{aligned}
& \underline{e}_{t+1}=\left[A_{0}-\underline{L} C\right] \underline{e}_{t}+\sum_{i=1}^{3} \underline{w}_{t}^{i}, \\
& \bar{e}_{t+1}=\left[A_{0}-\bar{L} C\right] \bar{e}_{t}+\sum_{i=1}^{3} \bar{w}_{i}^{t},
\end{aligned}
$$

where

$$
\begin{aligned}
\underline{w}_{t}^{1}= & \Delta A\left(\rho_{t}\right) x_{t}-\underline{\Delta A^{+}} \underline{x}_{t}^{+} \\
& +\overline{\Delta A} \underline{x}_{t}^{+}+\underline{\Delta A}^{-} \bar{x}_{t}^{+}-\overline{\Delta A} \bar{x}_{t}^{-}, \\
\underline{w}_{t}^{2}= & |\underline{\underline{L}}| V E_{p}-\underline{L}_{t}, \underline{w}_{t}^{3}=d_{t}-\underline{d}_{t} ; \\
\bar{w}_{t}^{1}= & \overline{\Delta A} \bar{x}_{t}^{+}-\underline{\Delta A} \bar{x}_{t}^{+} \\
& -\overline{\Delta A}{ }^{-} \underline{x}_{t}^{+}+\underline{\Delta A} \underline{x}_{t}^{-}-\Delta A\left(\rho_{t}\right) x_{t}, \\
\bar{w}_{t}^{2}= & \bar{L} v_{t}+|\bar{L}| V E_{p}, \bar{w}_{t}^{3}=\bar{d}_{t}-d_{t} .
\end{aligned}
$$


Let the gains $\underline{L}, \bar{L}$ be designed in order to verify (4.2) and suppose that $\underline{x}_{0} \leqslant x_{0} \leqslant \bar{x}_{0}$, then the dynamics for $\underline{e}_{t}, \bar{e}_{t}$ are cooperative and $\underline{e}_{0}, \bar{e}_{0} \in \mathbb{R}_{+}$, thus if $\underline{w}_{t}^{i} \geqslant 0, \bar{w}_{t}^{i} \geqslant 0$ for $i=\overline{1,3}$ and all $t \in \mathbb{N}_{+}$, then the relations (4.3) are satisfied. The inputs $\underline{w}_{t}^{i}, \bar{w}_{t}^{i}, i=2,3$ are nonnegative for all $t \in \mathbb{N}_{+}$due to Assumption 3.1. To prove that $\underline{w}_{t}^{1}$ and $\bar{w}_{t}^{1}$ remain nonnegative while the relation (4.3) is satisfied let us recall that by Assumption 3.2 the relations (3.3) are satisfied whereas the relation (4.3) is true, (3.3) implies that $\underline{w}_{t}^{1} \geqslant 0$ and $\bar{w}_{t}^{1} \geqslant 0$ for all $t \in \mathbb{N}_{+}$. However, the relation (4.3) is valid at time $t=0$ and it is preserved for all $t \in \mathbb{N}_{+}$by induction and cooperativity of dynamics of estimation errors $\underline{e}_{t}, \bar{e}_{t}$, therefore (4.3) and (3.3) are valid.

Let us show that the variables $\bar{x}_{t}$ and $\underline{x}_{t}$ stay bounded for all $t \in \mathbb{N}_{+}$in (3.1), (4.1). For this purpose let us rewrite the equations (4.1) as follows:

$$
\begin{aligned}
& \underline{x}_{t+1}=\left[A_{0}-\underline{L} C+\underline{\Delta A}^{+}\right] \underline{x}_{t}+\underline{f}\left(\underline{x}_{t}, \bar{x}_{t}\right)+\underline{\delta}_{t}, \\
& \bar{x}_{t+1}=\left[A_{0}-\bar{L} C+\overline{\Delta A}\right] \bar{x}_{t}+\bar{f}\left(\underline{x}_{t}, \bar{x}_{t}\right)+\bar{\delta}_{t},
\end{aligned}
$$

where

$$
\begin{gathered}
\underline{\delta}_{t}=\underline{L} y_{t}-|\underline{L}| V E_{p}+B u_{t}+\underline{d}_{t}, \\
\bar{\delta}_{t}=\bar{L} y_{t}+|\bar{L}| V E_{p}+B u_{t}+\bar{d}_{t}, \\
\underline{f}(\underline{x}, \bar{x})=\left(\underline{\Delta A}^{+}-\overline{\Delta A}\right) \underline{x}^{-}-\underline{\Delta A} \bar{x}^{+}+\overline{\Delta A} \bar{x}^{-}, \\
\bar{f}(\underline{x}, \bar{x})=\left(\overline{\Delta A}+\underline{\Delta A}^{+}\right) \bar{x}^{-}-\overline{\Delta A} \underline{x}^{+}+\underline{\Delta A^{-}} \underline{x}^{-} .
\end{gathered}
$$

The dynamics of $\underline{x}, \bar{x}$ in (4.5) are interrelated (both $\underline{f}$ and $\bar{f}$ depend on $\underline{x}$ and $\bar{x}$ ), however its linear part is nonnegative (the matrices $A_{0}-\underline{L} C+\underline{\Delta A^{+}}$and $A_{0}-\bar{L} C+\overline{\Delta A}^{+}$are nonnegative since $A_{0}-\underline{L} C, A_{0}-\bar{L} C$, $\underline{\Delta A}^{+}$and $\overline{\Delta A}$ are nonnegative ones), and the inputs $\underline{\delta}_{t}$ and $\bar{\delta}_{t}$ are bounded by Assumption 3.1 and the facts that $x \in \mathscr{L}_{\infty}^{n}, u \in \mathscr{L}_{\infty}^{m}$. The boundedness of $\underline{x}_{t}, \bar{x}_{t}$ is predefined by properties of the linear part and the functions $\underline{f}, \bar{f}$. Clearly $\underline{f}$ and $\bar{f}$ are globally Lipschitz. In addition, since $\left|\underline{x}^{-}\right| \leqslant|\underline{x}|,\left|\underline{x}^{+}\right| \leqslant|\underline{x}|$, $\left|\bar{x}^{-}\right| \leqslant|\bar{x}|$ and $\left|\bar{x}^{+}\right| \leqslant|\bar{x}|$, then

$$
\begin{aligned}
|\underline{f}(\underline{x}, \bar{x})| \leqslant & \left\|\underline{\Delta A}^{+}-\overline{\Delta A}\right\|_{2}|\underline{x}| \\
& +\left(\left\|\underline{\Delta A}^{-}\right\|_{2}+\|\overline{\Delta A}\|_{2}\right)|\bar{x}|, \\
|\bar{f}(\underline{x}, \bar{x})| \leqslant & \left.\left\|\overline{\Delta A}-\underline{\Delta A}^{+}\right\|\right|_{2}|\bar{x}| \\
& +\left(\|\overline{\Delta A}\|_{2}+\left\|\underline{\Delta A}^{-}\right\|_{2}\right)|\underline{x}| .
\end{aligned}
$$

To prove boundedness of the solutions of the observer (4.1), introduce the system

$$
\xi_{t+1}=G \xi_{t}+\phi\left(\xi_{t}\right)+\delta_{t}
$$

where

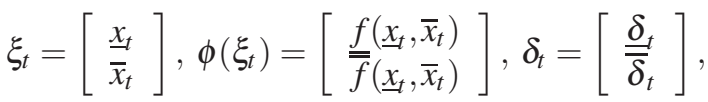

$$
\begin{aligned}
& \left|\phi\left(\xi_{t}\right)\right| \leqslant \eta\left|\xi_{t}\right|
\end{aligned}
$$

and $\delta \in \mathscr{L}_{\infty}^{2 n}$. Let us consider a Lyapunov function $V_{t}=\xi_{t}^{\mathrm{T}} P \xi_{t}$ (the matrix $P$ can be chosen diagonal 
since the matrix $G$ is nonnegative), whose increment takes the form:

$$
\begin{aligned}
& V_{t+1}-V_{t}=\xi_{t}^{\mathrm{T}}\left[G^{T} P G-P\right] \xi_{t}+\xi_{t}^{\mathrm{T}} G^{T} P \phi\left(\xi_{t}\right) \\
& +\phi^{T}\left(\xi_{t}\right) P G \xi_{t}+\phi^{T}\left(\xi_{t}\right) P \phi\left(\xi_{t}\right) \\
& +2 \xi_{t}^{\mathrm{T}} G^{T} P \delta_{t}+2 \delta_{t}^{\mathrm{T}} P \phi\left(\xi_{t}\right)+\delta_{t}^{\mathrm{T}} P \delta_{t} \\
& \leqslant \xi_{t}^{\mathrm{T}}\left[G^{T} P G-P\right] \xi_{t}+\xi_{t}^{\mathrm{T}} G^{T} P \phi\left(\xi_{t}\right) \\
& +\phi^{T}\left(\xi_{t}\right) P G \xi_{t}+\phi^{T}\left(\xi_{t}\right) P \phi\left(\xi_{t}\right) \\
& +2 \xi_{t}^{\mathrm{T}} G^{T} P \delta_{t}+2 \delta_{t}^{\mathrm{T}} P \phi\left(\xi_{t}\right)+ \\
& +\delta_{t}^{\mathrm{T}}[P+H-H] \delta_{t}+\gamma \eta^{2} \xi_{t}^{\mathrm{T}} \xi_{t} \\
& -\gamma \phi^{T}\left(\xi_{t}\right) \phi\left(\xi_{t}\right)+\xi_{t}^{\mathrm{T}} Q \xi_{t}-\xi_{t}^{\mathrm{T}} Q \xi_{t} \\
& \leqslant\left[\begin{array}{c}
\xi_{t} \\
\phi\left(\xi_{t}\right) \\
\delta_{t}
\end{array}\right]^{T} \Phi\left[\begin{array}{c}
\xi_{t} \\
\phi\left(\xi_{t}\right) \\
\delta_{t}
\end{array}\right]-\xi_{t}^{\mathrm{T}} Q \xi_{t} \\
& +\delta_{t}^{\mathrm{T}} H \delta_{t} \leqslant-\xi_{t}^{\mathrm{T}} Q \xi_{t}+\delta_{t}^{\mathrm{T}} H \delta_{t},
\end{aligned}
$$

due to (4.4). Thus, $\bar{x}_{t}$ and $\underline{x}_{t}$ stay bounded for all $t \in \mathbb{N}_{+}$(according to [17] the system is input-to-state stable from $\delta_{t}$ to $\xi_{t}$ ).

REMARK 4.1 By optimizing values of the constant $\gamma$ and the matrices $Q, H$ and $P$, it is possible to improve the accuracy of interval estimation since the signal $\delta_{t}$ represents the influence of uncertainty of the model (3.1), and the gain of the transfer from $\delta_{t}$ to $\xi_{t}$ characterizes the width of the interval $\left[\underline{x}_{t}, \bar{x}_{t}\right]$. This optimization problem has been considered in [33] for a class of continuous-time systems. A solution to the existence problem of interval observers with fast convergence and minimal $\mathscr{L}_{1}$-norm of the interval error has been proposed for deterministic systems. Some necessarily and sufficient conditions are checked by solving a linear programming problem. It has also been shown that uncertain systems can be considered in some cases. Recently, this framework has been investigated in [5] for the case of LPV continuous-time systems and a structure of interval observers and stability conditions have been studied in order to compute bounding solutions as accurate as possible. Optimization of the observer gains is addressed within the $\mathscr{L}_{1} / \mathscr{L}_{2}$ framework developped, for instance, in [4, 7]. Furthermore, in LMIs (4.9), (4.10) and (4.13), the matrices $Q, H, P$ and the scalar $\gamma$ are independent variables, which are not specified by a designer. They can be calculated by numerical solvers in order to satisfy these LMIs. Thus, the obtained values of these variables (and the observer gains $\underline{L}, \bar{L}$ ) are dependent on the used solver for LMIs and the version of software.

Due to diagonal structure of $P$, the conditions (4.2), (4.4) from Theorem 4.1 can be reformulated in terms of LMIs with respect to $\underline{L}, \bar{L}$ and $P$. Indeed, $G=D-\Lambda \Upsilon$ where

$$
\begin{gathered}
D=\left[\begin{array}{cc}
A_{0}+\underline{\Delta A^{+}} & 0 \\
0 & A_{0}+\overline{\Delta A}+
\end{array}\right], \Lambda=\left[\begin{array}{cc}
\underline{L} & 0 \\
0 & \bar{L}
\end{array}\right], \\
\Upsilon=\left[\begin{array}{cc}
C & 0 \\
0 & C
\end{array}\right] .
\end{gathered}
$$


We can rewrite the condition (4.4) as follows:

$$
\begin{gathered}
{\left[\begin{array}{ccc}
P-Q-\gamma \eta^{2} I_{2 n} & 0 & 0 \\
0 & \gamma I_{2 n} & 0 \\
0 & 0 & H
\end{array}\right]} \\
-\left[\begin{array}{c}
G^{T} P \\
P \\
P
\end{array}\right] P^{-1}\left[\begin{array}{c}
G^{T} P \\
P \\
P
\end{array}\right]^{T} \succeq 0 .
\end{gathered}
$$

Then introducing a new variable $W=P \Lambda(P G=P D-W \Upsilon)$ and using the Schur complement we derive an LMI that is equivalent to (4.4):

$$
\begin{aligned}
& {\left[\begin{array}{cccc}
P & P D-W \Upsilon & P & P \\
(P D-W \Upsilon)^{T} & P-Q-\gamma \eta^{2} I_{2 n} & 0 & 0 \\
P & 0 & \gamma I_{2 n} & 0 \\
P & 0 & 0 & H
\end{array}\right] \succeq 0} \\
& P \succ 0, Q \succ 0, H \succ 0,
\end{aligned}
$$

which has to be verified with a linear constraint corresponding to (4.2):

$$
P\left[\begin{array}{cc}
A_{0} & 0 \\
0 & A_{0}
\end{array}\right]-W \Upsilon \geqslant 0 .
$$

The matrix variable $P$ has to be declared diagonal, and $W$ has to be declared block-diagonal (with the same block structure as $\Lambda$ ), then these linear inequalities can be solved using a numerical routine (YALMIP toolbox of MATLAB [21], for instance, as it is done in the examples below).

REMARK 4.2 The requirement that the matrices $A_{0}-\underline{L} C, A_{0}-\bar{L} C$ have to be nonnegative can be relaxed by means of a change of coordinates $z=T x$ with a nonsingular matrix $T$ such that the matrices $T\left(A_{0}-\underline{L} C\right) T^{-1}, T\left(A_{0}-\bar{L} C\right) T^{-1}$ are nonnegative. The matrix $T$ can be found using the results of lemmas 2.3 (looking for $\underline{L}=\bar{L}=L$ ). This extension is omitted for brevity of presentation.

\subsection{Interval estimation and stabilization}

In this subsection the interval observer (4.1) is used in the design of a control law ensuring stabilization of (3.1). Thus the assumption on boundedness of $x \in \mathscr{L}_{\infty}^{n}$ or $u \in \mathscr{L}_{\infty}^{m}$ is no more needed, and only the relations (4.3) have to be ensured by a proper choice of the gains $\underline{L}, \bar{L}$. The control law to be designed should stabilize the interval observer, which due to (4.3) leads to stabilization of (3.1).

The main idea of the control synthesis is borrowed from [12]. According to Theorem 4.1, if in the observer (4.1) the gains $\underline{L}, \bar{L}$ are computed such that the matrices $A_{0}-\underline{L} C$ and $A_{0}-\bar{L} C$ are nonnegative, then the relations (4.3) are satisfied for any $u_{t} \in \mathbb{R}^{m}$. Therefore, ensuring stabilization of $\bar{x}_{t}, \underline{x}_{t}$ we provide a similar property for $x_{t}$, i.e. the problem of designing an output feedback for the stabilization of uncertain system (3.1) is replaced by the problem of a state feedback design for a completely known system (4.1). The only shortcoming, which we meet in this way, is that the dimension of the system (4.1) is twice larger than the dimension of (3.1), but the control dimension is not changed. Furthermore, since the interval observer (4.1) is composed of two classical ones, an alternative control law can be built under differing assumptions by using only the lower or the upper bound $(\underline{x}, \bar{x})$ as suggested in [26]. 
THEOREM 4.2 Let assumptions 3.1, 3.2 be satisfied and given a domain of the initial conditions such that $\underline{x}_{0} \leqslant x_{0} \leqslant \bar{x}_{0}$. Let

$$
u_{t}=\underline{K x_{t}}+\bar{K} \bar{x}_{t}
$$

where $\underline{L}, \bar{L}, \underline{K} \in \mathbb{R}^{m \times n}$ and $\bar{K} \in \mathbb{R}^{m \times n}$ are selected such that assumption 4.1 is satisfied and there exist matrices $P \in \mathbb{R}^{2 n \times 2 n}, P=P^{T} \succ 0, Q \in \mathbb{R}^{2 n \times 2 n}, Q=Q^{T} \succ 0, H \in \mathbb{R}^{2 n \times 2 n}, H=H^{T} \succ 0$ and a constant $\gamma>$ 0 such that the matrix inequality (4.4) is satisfied, where $\eta=\sqrt{2}\left(\left\|\underline{\Delta A^{+}}-\overline{\Delta A}\right\|_{2}+\left\|\overline{\Delta A}-\underline{\Delta A}^{-}\right\|_{2}+\right.$ $\left.\sqrt{n} \max \left\{\|\underline{L} C\|_{2},\|\bar{L} C\|_{2}\right\}\right)$ and

$$
G=\left[\begin{array}{cc}
A_{0}-\underline{L C}+\underline{\Delta A}^{+}+B \underline{K} & B \bar{K}-\underline{\Delta A}^{-} \\
B \underline{K}-\overline{\Delta A}^{-} & A_{0}-\bar{L} C+\overline{\Delta A}^{+}+B \bar{K}
\end{array}\right] .
$$

Then, the relation (4.3) is verified and

$$
\begin{aligned}
\left|x_{t}\right| \leqslant & \sqrt{n}\left|\left[\begin{array}{c}
\underline{x}_{t} \\
\bar{x}_{t}
\end{array}\right]\right| \leqslant \sqrt{\frac{n}{\lambda_{\min }(P)}}\left(\sqrt{\lambda_{\max }(P)} \alpha^{0.5 t}\left(\left|\underline{x}_{0}\right|+\left|\bar{x}_{0}\right|\right)\right. \\
& \left.+\sqrt{\frac{\lambda_{\max }(H)}{1-\alpha}}\left[\|\underline{d}\|+\|\bar{d}\|+2\left(\|\underline{L}\|_{2}+\|\bar{L}\|_{2}\right) V\right]\right),
\end{aligned}
$$

where $\alpha=1-\lambda_{\min }(Q) / \lambda_{\max }(P)$.

Proof. The proof that the relations (4.3) are satisfied under (4.2) and assumptions 3.1, 3.2 is the same as in Theorem 4.1. Let us show that for the control (4.11) all solutions of (4.1) are bounded if the condition (4.4) is verified (due to (4.3) the solutions of (3.1) will also be bounded in this case).

Let us substitute (4.11) in (4.1), then we obtain

$$
\begin{aligned}
& \underline{x}_{t+1}=\left[A_{0}-\underline{L} C+\underline{\Delta A}^{+}+B \underline{K}\right] \underline{x}_{t}+\left(B \bar{K}-\underline{\Delta A^{-}}\right) \bar{x}_{t} \\
& +\underline{f}\left(\underline{x}_{t}, \bar{x}_{t}, x_{t}\right)+\underline{\delta}_{t}, \\
& \bar{x}_{t+1}=\left[A_{0}-\bar{L} C+\overline{\Delta A}^{+}+B \bar{K}\right] \bar{x}_{t}+\left(B \underline{K}-\overline{\Delta A}^{-}\right) \underline{x}_{t} \\
& +\bar{f}\left(\underline{x}_{t}, \bar{x}_{t}, x_{t}\right)+\bar{\delta}_{t}, \\
& \underline{f}\left(\underline{x}_{t}, \bar{x}_{t}, x_{t}\right)=\left[\left(\underline{\Delta A}^{+}-\overline{\Delta A}^{+}\right) \underline{x}_{t}^{-}+\left(\overline{\Delta A}^{-}\right.\right. \\
& \left.\left.-\underline{\Delta A^{-}}\right) \bar{x}_{t}^{-}\right]+\underline{L} C x_{t}, \\
& \bar{f}\left(\underline{x}_{t}, \bar{x}_{t}, x_{t}\right)=\left[\left(\overline{\Delta A}^{+}-\underline{\Delta A}^{+}\right) \bar{x}_{t}^{-}+\left(\underline{\Delta A}^{-}\right.\right. \\
& \left.-\overline{\Delta A}) \underline{x}_{t}^{-}\right]+\bar{L} C x_{t}, \\
& \underline{\delta}_{t}=\underline{d}_{t}+\underline{L} v_{t}-|\underline{L}| V E_{p}, \bar{\delta}_{t}=\bar{d}_{t}+\bar{L} v_{t}+|\bar{L}| V E_{p} \text {, }
\end{aligned}
$$

where $\underline{\delta}, \bar{\delta} \in \mathscr{L}_{\infty}^{n}$ due to Assumption 3.1. Note that for the stabilization problem (without the assumption that $x \in \mathscr{L}_{\infty}^{n}$ ) from (4.3) we have

$$
\begin{aligned}
\left|x_{t}\right|_{\infty} & \leqslant \max \left\{\left|\underline{x}_{t}\right|_{\infty},\left|\bar{x}_{t}\right|_{\infty}\right\} \quad \forall t \in \mathbb{N}_{+}, \\
\left|x_{t}\right| & \leqslant \sqrt{n}\left|x_{t}\right|_{\infty} \leqslant \sqrt{n} \max \left\{\left|\underline{x}_{t}\right|_{\infty},\left|\bar{x}_{t}\right|_{\infty}\right\} \\
& \leqslant \sqrt{n} \max \left\{\left|\underline{x}_{t}\right|,\left|\bar{x}_{t}\right|\right\}
\end{aligned}
$$


10 of 16

then $\underline{f}$ and $\bar{f}$ are globally Lipschitz in $\bar{x}_{t}$ and $\underline{x}_{t}$. For the stability analysis, introduce an auxiliary system:

$$
\begin{aligned}
& \xi_{t+1}=G \xi_{t}+\phi\left(\xi_{t}, x_{t}\right)+\delta_{t}, \\
& \xi_{t}=\left[\begin{array}{c}
\underline{x}_{t} \\
\bar{x}_{t}
\end{array}\right], \phi\left(\xi_{t}, x_{t}\right)=\left[\begin{array}{c}
f\left(\underline{x}_{t}, \bar{x}_{t}, x_{t}\right) \\
\bar{f}\left(\underline{x}_{t}, \bar{x}_{t}, x_{t}\right)
\end{array}\right], \delta_{t}=\left[\begin{array}{c}
\underline{\delta}_{t} \\
\bar{\delta}_{t}
\end{array}\right],
\end{aligned}
$$

$\left|\phi\left(\xi_{t}, x_{t}\right)\right| \leqslant \eta\left|\xi_{t}\right|$ and $\delta \in \mathscr{L}_{\infty}^{2 n}$. Let us consider a Lyapunov function $V_{t}=\xi_{t}^{\mathrm{T}} P \xi_{t}$, whose increment takes the form (4.8) for the matrix $G$ given in Theorem 4.2 and under substitution $\phi\left(\xi_{t}, x_{t}\right) \rightarrow \phi\left(\xi_{t}\right)$. Therefore,

$$
V_{t+1} \leqslant \alpha V_{t}+\lambda_{\max }(H)\left|\delta_{t}\right|^{2}
$$

for all $t \in \mathbb{N}_{+}$and $|\alpha|<1$ by definition of $Q$ and $P$, then $\left|x_{t}\right| \leqslant \sqrt{n}\left|\xi_{t}\right|$ and the estimation of the rate of decreasing for $x_{t}, \bar{x}_{t}$ and $\underline{x}_{t}$ given in Theorem 4.2 follows.

REMARK 4.3 Note that if $V=0$ (there is no measurement noise) and $\underline{d}_{t}=\bar{d}_{t}=0$ for all $t \in \mathbb{N}_{+}$(no additive disturbance), then in this case the system uncertainty is presented by the term $\Delta A\left(\rho_{t}\right) x_{t}$ only, and the interval observer-based control (4.1), (4.11) provides global asymptotic stability property of the LPV system (3.1).

The conditions for $\underline{L}, \bar{L}$ are given by (4.2), and that for the control gains $\underline{K}, \bar{K}$ by (4.4), thus the conditions for the observer gains $\underline{L}, \bar{L}$ and the controller gains $\underline{K}, \bar{K}$ are not independent, but they can be solved consequently. Note that in order to simplify a solution of (4.4) with respect to $\underline{K}, \bar{K}$ we can add some additional constraints to (4.2) for the design of $\underline{L}, \bar{L}$. For example, we can ask for Schur stability of $A_{0}-\underline{L} C+\underline{\Delta A^{+}}, A_{0}-\bar{L} C+\overline{\Delta A}^{+}$or the matrix $G$ under substitution $\underline{K}=\bar{K}=0$.

In particular, the following LMIs can be formulated to verify an extended condition (4.2) for the gains $\underline{L}, \bar{L}$ :

$$
\begin{gathered}
{\left[\begin{array}{cc}
{\left[A_{0}+\underline{\Delta A}^{+}\right)^{T} \underline{P}-C^{T} \underline{W}^{T}} & \underline{P}\left(A_{0}+\underline{\Delta A}^{+}\right)-\underline{W} C \\
\bar{P} & \succ 0, \\
\left.\left(A_{0}+\overline{\Delta A}\right)^{T}\right)^{T}-C^{T} \bar{W}^{T} & \bar{P}\left(A_{0}+\overline{\Delta A}^{+}\right)-\bar{W} C \\
\underline{P} A_{0}-\underline{W} C \geqslant 0, \bar{P} A_{0}-\bar{W} C \geqslant 0, \underline{P} \succ 0, \bar{P} \succ 0,
\end{array}\right] \succ 0,}
\end{gathered}
$$

which have to be solved with respect to diagonal matrices $\underline{P} \in \mathbb{R}_{+}^{n \times n}, \bar{P} \in \mathbb{R}_{+}^{n \times n}$ and some matrix variables $\underline{W} \in \mathbb{R}^{n \times p}, \bar{W} \in \mathbb{R}^{n \times p}$, then $\underline{L}=\underline{P}^{-1} \underline{W}, \bar{L}=\bar{P}^{-1} \bar{W}$. These LMIs imply (4.2) and Schur stability of $A_{0}-\underline{L} C+\underline{\Delta A^{+}}, A_{0}-\bar{L} C+\overline{\Delta A}$. In order to rewrite the inequality (4.4) in a form suitable for numerical computation, let us define

$$
\begin{gathered}
D=\left[\begin{array}{cc}
A_{0}-\underline{L} C+\underline{\Delta A}^{+} & -\underline{\Delta A^{-}} \\
-\overline{\Delta A} & A_{0}-\bar{L} C+\overline{\Delta A}+
\end{array}\right], \\
\Xi=\left[\begin{array}{c}
B \\
B
\end{array}\right], K=[\underline{K} \bar{K}],
\end{gathered}
$$


then $G=D+\Xi K$. Using Schur complement (similarly as in the previous subsection) we obtain

$$
\begin{aligned}
& {\left[\begin{array}{cccc}
P & P G & P & P \\
G^{T} P & P-Q-\gamma \eta^{2} I_{2 n} & 0 & 0 \\
P & 0 & \gamma I_{2 n} & 0 \\
P & 0 & 0 & H
\end{array}\right] \succeq 0} \\
& P \succ 0, Q \succ 0, H \succ 0,
\end{aligned}
$$

next multiplying this inequality from the left and from the right by a symmetric matrix $\operatorname{diag}\left[P^{-1}, P^{-1}, I_{2 n}, I_{2 n}\right]$ we get for $S=P^{-1}$ :

$$
\begin{aligned}
& {\left[\begin{array}{cccc}
S & G S & I_{2 n} & I_{2 n} \\
S G^{T} & S-Z-\gamma \eta^{2} S^{2} & 0 & 0 \\
I_{2 n} & 0 & \gamma I_{2 n} & 0 \\
I_{2 n} & 0 & 0 & H
\end{array}\right] \succeq 0} \\
& S \succ 0, Z \succ 0, H \succ 0 \text {, }
\end{aligned}
$$

where $Z=P^{-1} Q P^{-1} \in \mathbb{R}^{2 n \times 2 n}$ and $S \in \mathbb{R}^{2 n \times 2 n}$ are new variables. Applying again Schur complement to the term $-\gamma \eta^{2} S^{2}$ we can rewrite the last LMIs as follows for a new variable $M \in \mathbb{R}^{m \times 2 n}$ :

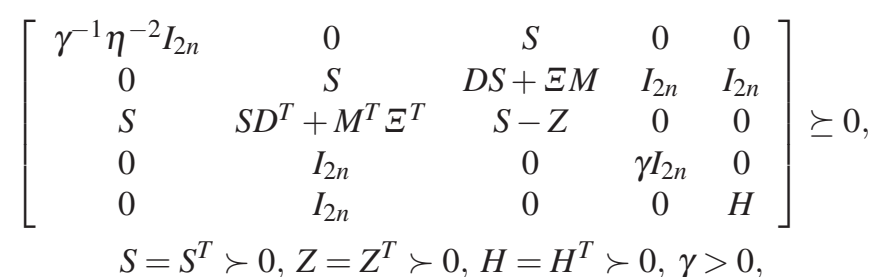

then $K=M S^{-1}$.

\section{Examples}

In this section, we consider an academic example to discuss the validity of conditions of theorems 4.1 and 4.2:

$$
\begin{gathered}
A_{0}=\frac{1}{10}\left[\begin{array}{ccc}
0 & 1 & 3 \\
0 & 8 & 2 \\
-0.1 & 0 & 8
\end{array}\right], B=\left[\begin{array}{l}
0 \\
0 \\
1
\end{array}\right], C=\left[\begin{array}{l}
1 \\
0 \\
0
\end{array}\right]^{T} \\
\overline{\Delta A}=0.015\left[\begin{array}{ccc}
0.1 & 1 & 1 \\
0.1 & 0.1 & 1 \\
0.1 & 0.1 & 1
\end{array}\right], \underline{\Delta A}=-\overline{\Delta A} \\
V=0.2, v_{t}=V \sin (t) \\
d_{t}=\left[\begin{array}{l}
1 \\
1 \\
0
\end{array}\right] \sin (C x)+e_{t},\|e\| \leqslant \varepsilon=0.1 .
\end{gathered}
$$


For simulation we selected

$$
\begin{gathered}
\Delta A\left(\rho_{t}\right)=0.015 \\
\times\left[\begin{array}{ccc}
0.1 \sin \left(\omega_{1} t\right) & \sin \left(\omega_{2} t\right) & \cos \left(\omega_{1} t\right) \\
\cos \left(\omega_{2} t\right) & 0.1 \sin \left(2 \omega_{1} t\right) & \cos \left(2 \omega_{1} t\right) \\
0.1 \sin \left(\omega_{1} t / 2\right) & 0.1 \cos \left(\omega_{2} t / 2\right) & \sin \left(\omega_{1} t\right) \cos \left(\omega_{2} t\right)
\end{array}\right], \\
e_{t}=\varepsilon\left[\begin{array}{c}
\sin \left(\omega_{2} t\right) \\
\sin \left(\omega_{1} t\right) \\
\cos \left(\omega_{2} t\right)
\end{array}\right], \omega_{1}=0.2, \omega_{2}=1 / 3 .
\end{gathered}
$$

The matrix $A_{0}$ has a negative element, and this system is not asymptotically stable for $u_{t}=0$ at the origin.

Consider the problem of interval estimation using Theorem 4.1, then

$$
\underline{L}=\bar{L}=\left[\begin{array}{lll}
0 & 0 & 0.01
\end{array}\right]^{T}
$$

is a solution of LMIs (4.9), (4.10) with

$$
P=\operatorname{diag}[0.02880 .14490 .36190 .030 .14790 .4048] \text {. }
$$

The results of the system simulation for $u_{t}=-y_{t}$ are given on Fig. 1 (on the left). As we can conclude from the results of simulation, the accuracy of the estimation for the variable $x_{3}$ is better than for $x_{2}$ since the equation for $x_{3}$ does not contain the disturbance $d$.

In addition, to illustrate the performances of the proposed methodology, consider the prediction/correction estimator based on interval arithmetics [16]. Similarly, the initial state is assumed to belong into an interval and the same bounds are used for noises and disturbances. The prediction consists in evaluating at each time instant the interval $\left[x_{t+1}\right]^{+}$which is consistent with $\left[x_{t}\right]$ and with the state equation. Thus, $\left[x_{t+1}\right]^{+}$is computed by interval arithmetics through:

$$
\left[x_{t+1}\right]^{+}=\left[A\left(\rho_{t}\right)\right]\left[x_{t}\right]+B u_{t}+\left[d_{t}\right],
$$

where $\left[A\left(\rho_{t}\right)\right]=\left[A_{0}+\Delta A\left(\rho_{t}\right)\right]=A_{0}+[-\underline{\Delta A}, \overline{\Delta A}]$.

At the time instant $t+1$, the measurement $y_{t+1}$ becomes available. Since it is subject to a bounded noise $v\left(v_{t} \in\left[\underline{v}_{t}, \bar{v}_{t}\right]\right)$, the output belongs into an interval $\left[y_{t+1}\right]=\left[y_{t+1}-V, y_{t+1}+V\right]$. The correction consists in contracting the predicted interval $\left[x_{t+1}\right]^{+}$by using the new information. Denote by $\mathbb{X}_{t+1}^{*}$, the set of all the state values which are consistent with $\left[y_{t+1}\right]$. It is defined by $\mathbb{X}_{t+1}^{*}=\left\{x \in \mathbb{R}^{n} \mid, C x \in\right.$ $\left.\left[y_{t+1}\right]\right\}$ and is often given by a polytope which has to be outer approximated, at the cost of an additional conservatism, by a box $\left[x_{t+1}\right]^{*}$. Finally, the estimated state box at $t+1$ is given by:

$$
\left[x_{t+1}\right]=\left[x_{t+1}\right]^{+} \cap\left[x_{t+1}\right]^{*} .
$$

For the considered numerical example, the results of simulations with the proposed approach and those computed by the prediction/correction estimator are plotted in Fig 1 (on the right).

The simulations show clearly that the prediction/correction estimator generates more conservative results (larger intervals). Instead of outer approximating the feasible state set, subpavings (union of intervals) are used in [16]. The idea is to split the initial state box $\left[x_{0}\right]$ into $N$ smaller $\left[x_{0}\right]_{i}, i=1 \ldots N$, and the prediction scheme (5.1) is applied to each of these sub-boxes. Furthermore, the predicted subboxes should be merged in one box in order to perform the correction. The splitting and merging should be performed at each time instant. Therefore, the latter procedure can be time-consuming. 

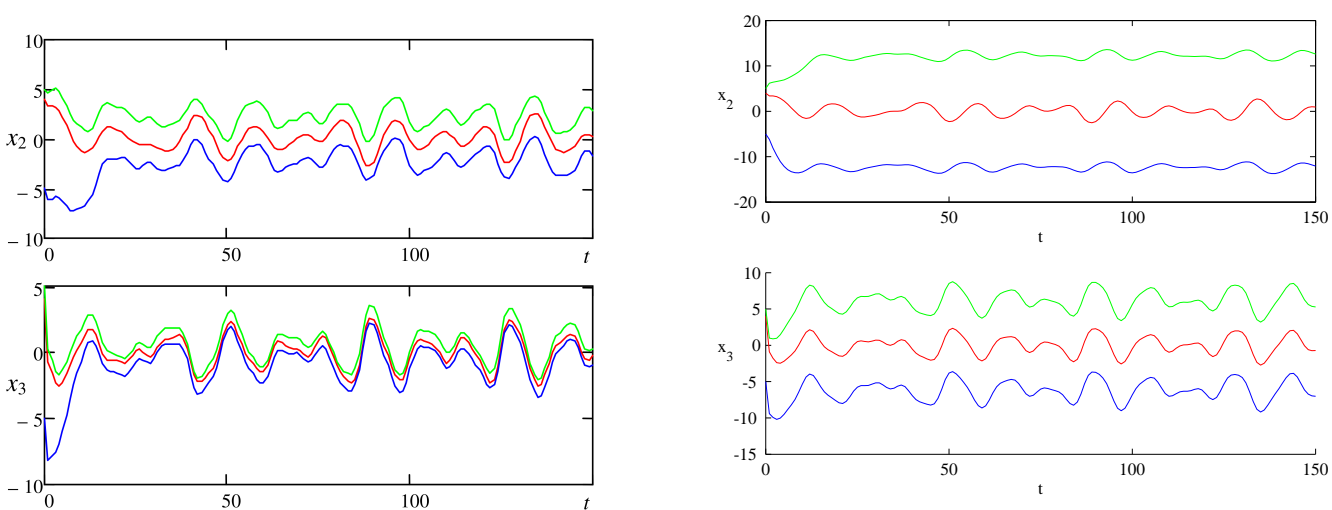

FIG. 1. The results of simulations for the case of estimation. Proposed approach on the left. Prediction/correction on the right.
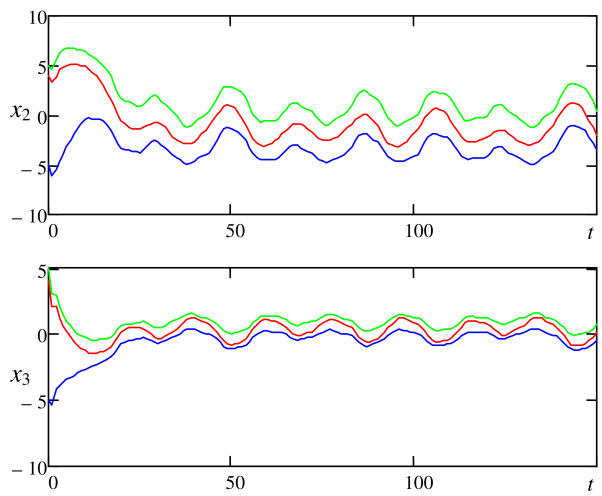

FIG. 2. The results of simulations for the case of estimation and stabilization

Next, consider the problem of output stabilization from Theorem 4.2, when the same $\underline{L}, \bar{L}$ are solutions of LMI (4.12) and

$$
\begin{aligned}
\underline{K} & =[0-0.1765-0.3647], \\
\bar{K} & =[-0.0004-0.2409-0.544]
\end{aligned}
$$

is a solution of LMI (4.13). The results of estimation and stabilization of the system with control (4.11) are given in Fig. 2. As we can see from comparison of figures 1 and 2, the state feedback in the case of Fig. 2 provides better quality of regulation and disturbance attenuation than the output one from Fig. 1. The accuracy of interval estimation is again better for $x_{3}$.

\section{Conclusion}

The problems of interval state estimation and robust stabilization are studied for discrete-time LPV systems with unmeasurable vector of scheduling parameters. For solution of both problems different 
conditions of cooperativity and stability are expressed in terms of LMIs. The efficiency of the proposed observers is demonstrated on numerical simulations. The optimization of the observer gains, for instance within the $\mathscr{L}_{1} / \mathscr{L}_{2}$ framework [3,8], is the subject of further works in order to improve the accuracy of interval estimation.

\section{References}

[1] T. Alamo, J.M. Bravo, and E.F. Camacho. Guaranteed state estimation by zonotopes. Automatica, 41(6):1035-1043, 2005.

[2] O. Bernard and J.L. Gouzé. Closed loop observers bundle for uncertain biotechnological models. Journal of Process Control, 14(7):765-774, 2004.

[3] C. Briat. Robust stability analysis of uncertain linear positive systems via integral linear constraints: L1- and linfty-gain characterizations. In Proc. 50th IEEE CDC and ECC, pages 63376342, Orlando, 2011.

[4] C. Briat. Robust stability analysis of uncertain linear positive systems via integral linear constraints: $l_{1}$ and $l_{\infty}$ gain characterizations. In 50th IEEE Conference on Decision and Control and European Control Conference, pages 6337-6342, Dec 2011.

[5] S. Chebotarev, D. Efimov, T. Raïssi, and A. Zolghadri. Interval observers for continuous-time LPV systems with $l_{1} / l_{2}$ performance. Automatica, 51(7), 2015.

[6] C. Combastel. Stable interval observers in bbc for linear systems with time-varying input bounds. IEEE Transactions on Automatic Control, 58(2):481-487, 2013.

[7] Y. Ebihara, D. Peaucelle, and D. Arzelier. $l_{1}$ gain analysis of linear positive systems and its application. In 50th IEEE Conference on Decision and Control and European Control Conference, pages 4029-4034, Dec 2011.

[8] Y. Ebihara, D. Peaucelle, and D. Arzelier. L1 gain analysis of linear positive systems and its application. In Proc. 50th IEEE CDC and ECC, pages 4029-4035, Orlando, 2011.

[9] D. Efimov, L.M. Fridman, T. Raïssi, A. Zolghadri, and R. Seydou. Interval estimation for LPV systems applying high order sliding mode techniques. Automatica, 48:2365-2371, 2012.

[10] D. Efimov, W. Perruquetti, T. Raïssi, and A. Zolghadri. On interval observer design for discrete systems. In Proc. ECC 2013,, 2013.

[11] D. Efimov, T. Raïssi, S. Chebotarev, and A. Zolghadri. Interval state observer for nonlinear timevarying systems. Automatica, 49(1):200-205, 2013.

[12] D. Efimov, T. Raïssi, and A. Zolghadri. Control of nonlinear and LPV systems: interval observerbased framework. IEEE Trans. Automatic Control, 58(3):773-782, 2013.

[13] L. Farina and S. Rinaldi. Positive Linear Systems: Theory and Applications. Wiley, New York, 2000.

[14] M. W. Hirsch and H. L. Smith. Monotone maps: a review. J. Difference Equ. Appl., 11(4-5):379$398,2005$. 
[15] L. Jaulin. Nonlinear bounded-error state estimation of continuous-time systems. Automatica, 38(2):1079-1082, 2002.

[16] L. Jaulin, M. Kieffer, O. Didrit, and E. Walter. Applied interval analysis. Springer, London, 2001.

[17] Z.P. Jiang and Y. Wang. Input-to-state stability for discrete-time nonlinear systems. Automatica, 37(6):857-869, 2001.

[18] M. Kieffer and E. Walter. Guaranteed nonlinear state estimator for cooperative systems. Numerical Algorithms, 37:187-198, 2004.

[19] I.E. Kose and F. Jabbari. Control of LPV systems with partly measured parameters. Automatic Control, IEEE Transactions on, 44(3):658-663, Mar 1999.

[20] M. Krstić and H. Deng. Stabilization of nonlinear uncertain systems. Communications and Control Engineering. Springer, 1998.

[21] J. Löfberg. Automatic robust convex programming. Optimization methods and software, 27(1):115-129, 2012.

[22] A. Marcos and J. Balas. Development of linear parameter-varying models for aircraft. J. Guidance, Control, Dynamics, 27(2):218-228, 2004.

[23] F. Mazenc and O. Bernard. Interval observers for linear time-invariant systems with disturbances. Automatica, 47(1):140-147, 2011.

[24] F. Mazenc, T. N. Dinh, and S. I. Niculescu. Interval observers for discrete-time systems. In Proc. IEEE CDC 2012, 2012.

[25] F. Mazenc, T.N. Dinh, and S.I. Niculescu. Interval observers for discrete-time systems. International Journal of Robust and Nonlinear Control, 2013.

[26] F. Mazenc, T.N. Dinh, and S.I. Niculescu. Robust interval observers and stabilization design for discrete-time systems with input and output. Automatica, 49(11):3490 - 3497, 2013.

[27] J. Mohammadpour and C. W. Scherer, editors. Control of Linear Parameter Varying Systems with Applications. Springer, 2012.

[28] M. Moisan, O. Bernard, and J.L. Gouzé. Near optimal interval observers bundle for uncertain bio-reactors. Automatica, 45(1):291-295, 2009.

[29] B. T. Polyak, S. A. Nazin, C. Durieu, and E. Walter. Ellipsoidal parameter or state estimation under model uncertainty. Automatica, 40(7):1171-1179, 2004.

[30] T. Raïssi, D. Efimov, and A. Zolghadri. Interval state estimation for a class of nonlinear systems. IEEE Trans. Automatic Control, 57(1):260-265, 2012.

[31] T. Raïssi, N. Ramdani, and Y. Candau. Set membership state and parametre estimation for systems described by nonlilear differential equations. Automatica, 40:1771-1777, 2004.

[32] T. Raïssi, G. Videau, and A. Zolghadri. Interval observers design for consistency checks of nonlinear continuous-time systems. Automatica, 46(3):518-527, 2010. 
[33] M.A. Rami, C.H. Cheng, and C. de Prada. Tight robust interval observers: An lp approach. In Decision and Control, 2008. CDC 2008. 47th IEEE Conference on, pages 2967-2972, 2008.

[34] J. Shamma and J. Cloutier. Gain-scheduled missile autopilot design using linear parameter-varying transformations. J. Guidance, Control, Dynamics, 16(2):256-261, 1993.

[35] J.S. Shamma. Control of Linear Parameter Varying Systems with Applications, chapter An overview of LPV systems, pages 1-22. Springer, 2012.

[36] W. Tan. Applications of Linear Parameter-Varying Control Theory. PhD thesis, Dept. of Mechanical Engineering, University of California at Berkeley, 1997. 